\title{
ULTRAVIOLET IMAGING OF THE JOVIAN AURORA WITH THE HUBBLE SPACE TELESCOPE
}

\author{
V. Dols ${ }^{1}$, J.C. Gérard ${ }^{1}$, F. Paresce ${ }^{2}$, R. Prangé ${ }^{3,4}$ and A. Vidal-Madjar ${ }^{4}$
}

\begin{abstract}
We present here for the first time a Lyman$\alpha$ image of the north polar region of Jupiter obtained with the Faint Object Camera (FOC) on board the Hubble Space Telescope a few hours after the encounter of the ULYSSES spacecraft with Jupiter. The presence of high latitude regions of enhanced emission is clearly observed. A comparison with the location of the "UVS oval", the Io $(\mathrm{L}=6)$ and highlatitude field-line footprints shows that the best agreement is obtained with the $L \geq 15$ footprint and the UVS oval which are close to each other for the particular longitudinal sector $\left(30^{\circ}<\lambda_{I I I}<210^{\circ}\right)$ observed. These two L-shells correspond to two possible sources of precipitation: particles originating respectively from the region of the plasma torus of Io in a distorted magnetic field or particles from the distant magnetosphere by analogy with the terrestrial aurora. The first direct determination of the latitudinal extent of the oval and of its intensity is made and compared with previous estimates.
\end{abstract}

\section{Introduction}

Observations made with the Voyager 1 Ultraviolet Spectrometer (UVS) in 1979 (Broadfoot et al., 1979) and subsequent spectrometric measurements (Herbert et al., 1987) obtained with the Voyager 2 and International Ultraviolet Explorer (IUE) (Clarke et al., 1980) identified the presence of strong auroral emissions due to $\mathrm{H}_{2}$ and HI Lyman- $\alpha$ distributed around the poles of Jupiter. Meridional scans of the north $\mathrm{H}_{2}$ UV aurora with the UVS slit were used to define the equatorial boundary of the oval ("the UVS oval") which appeared to lie close to but not coincident with the lo orbit field line footprint (Broadfoot et al., 1981). A longitudinal variation of the north intensity with $\mathrm{H}_{2}$ and Lyman- $\alpha$ peaks generally centered near a longitude $\lambda_{I I I}=180^{\circ}$ was observed both during the Voyager encounters (Herbert et al., 1987) and IUE long-term survey (Livengood et al., 1990). However, the real morphology of the FUV aurora, which bears the signature of the auroral precipitation processes and more generally of the magnetosphere dynamics, was still basically unknown due to the lack of any imaging instrument and to the limited spatial resolution of most of the observations made so far. The first image at Lyman- $\alpha$ has been recorded with the Faint Object Camera (FOC) on board the Hubble Space Telescope (HST) at the time of the ULYSSES Jupiter fly-by and we describe here its preliminary analysis.

\section{Instrumental configuration}

The image of the Jovian north polar region described below is a 16-minute exposure obtained at 02:58 UT on February

\footnotetext{
${ }^{1}$ Institut d'Astrophysique - Université de Liège, Belgium

${ }^{2}$ Space Telescope Science Institute, Baltimore, USA

${ }^{3}$ Institut d'Astrophysique Spatiale, Orsay, France

${ }^{4}$ Institut d'AAstrophysique, Paris, France
}

\section{Copyright 1992 by the American Geophysical Union.}

Paper number 92GL02104

0094-8534/92/92GL-02104\$03.00
9, 1992 about 15 hours after the closest approach of the ULYSSES spacecraft with Jupiter. The F/96 optical relay of the FOC was used with the F120M + F140W filter combination. The FOC and the filter characteristics are described in detail by Paresce (1992). During the observations, Jupiter was at a distance of 4.47 AU from the Earth, the phase angle was $4.2^{\circ}$ and the latitude of the sub-Earth point $1.72^{\circ}$ $S$. The angular equatorial diameter of the planet was 43.94 arcsec. Consequently only a fraction of the northern hemisphere could be imaged in the 22 arcsec $\times 22$ arcsec field of view of the camera. The image was obtained in the 1024 $\times 512$ zoomed pixel format with $22 \times 44$ milliarcsec pixels projecting onto a $143 \times 286 \mathrm{~km}^{2}$ area on the planet at $60^{\circ}$. The planet was tracked using the latest possible ephemeris for the spacecraft and the north pole was kept at the center of the detector during the exposure. The possible enror on the position of the planet in the FOC field of view is of the order of 1 arcsec whereas the tracking error is less than 0.02 arcsec. The central meridian longitude $\lambda_{I I}(1965)$ was $115^{\circ}$ and the Io orbital phase angle was $96^{\circ} \mathrm{W}$, measured from inferior geocentric conjunction (sub-Earth meridian) that is close to maximum western elongation.

The F120M + F140W combination of filters was selected to isolate the HI Lyman- $\alpha$ emission while rejecting efficiently the other light components due mainly to the $\mathrm{H}_{2}$ auroral and airglow UV emission and sunlight scattered by the planetary atmosphere. The use of two UV filters in series is necessary to remove the contamination due to near-UV and visible light otherwise transmitted in the long-wavelength wing of a single UV filter. This filter combination has an approximately gaussian transmission profile reaching $0.65 \%$ at the peak $(1250 \mathrm{~A})$ and $0.42 \%$ at $1216{ }^{\circ} \mathrm{A}$ with a full width at half maximum (FWHM) of $90 \mathrm{~A}$. The intensity contribution of the hydrogen bands has been determined by integrating the intensity distribution calculated with a $\mathrm{H}_{2}$ synthetic spectrum over the effective passband of the filters. The synthetic spectrum is calculated using the Lyman/Werner and vibrational intensity ratios based on the study by Ajello et al. (1984) which applies to electrons of $\sim 100 \mathrm{eV}$ and we assume no hydrocarbon absorption. Using a typical value of 1.3 for the Jovian $\mathrm{H}_{2}$-bands/Lyman $\alpha$ auroral ratio (Sandel et al., 1979), we estimate the relative contribution of the $\mathrm{H}_{2}$ Lyman and Werner bands in the auroral signal to about $28 \%$ of the total. The combined efficiency of the filters, telescope optical elements and detector is low and the observed count rate is thus small. A maximum of about $1 \times 10^{-3} \mathrm{C} \mathrm{s}^{-1}$ pix $^{-1}$ obtained, well below the threshold of non linearity of the detector of $0.3 \mathrm{C} \mathrm{s}^{-1} \mathrm{pix}^{-1}$. The raw image was flattened and geometrically corrected to remove the optical and detector distortions. The spherical aberration of the primary mirror of the HST spreads the light of a point source over a circular halo of about 2 arcsec radius. The encircled energy within a 0.1 arcsec of the central peak is about $15 \%$ of the total energy. As a consequence of the low count rate, standard restoration techniques cannot be applied in this case to improve the image quality. Instead, smoothing and rebinning on a number of pixels were applied to improve the signal/noise ratio.

\section{Lyman- $\alpha$ image of the north jovian aurora}

Figure 1 shows the corrected image with an effective 28 $\times 14$ pixel size $(0.62 \operatorname{arcsec} \times 0.62$ arcsec $)$ and an overlaid grid of meridians and parallels taking the oblateness of the 


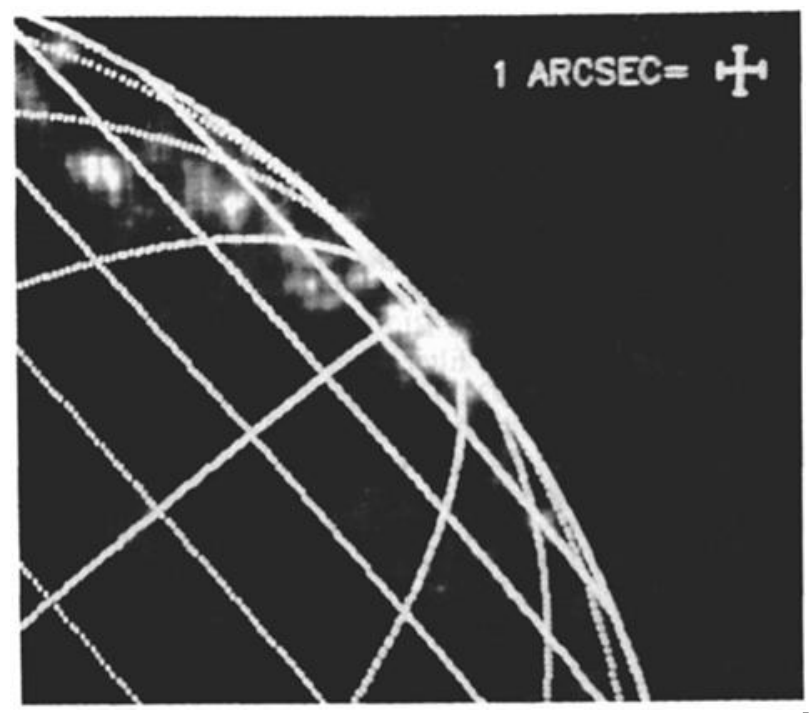

Figure 1: false colour image of the northern polar region of Jupiter observed with the FOC on February 9, 1992. The $\lambda_{I I I}$ longitude of the central meridian is $115^{\circ}$ and meridians increase by steps of $20^{\circ}$ toward the upper left corner. The bright feature extending from the vicinity of the north pole to the eastern (astronomical) limb is interpreted as the Lyman- $\alpha$ signature of the Jovian aurora excited by energetic particle precipitation. planet into consideration. A roughly continuous region of enhanced intensity is clearly observed extending from the vicinity of the rotational north pole to the (astronomical) eastern limb near a latitude of $55^{\circ}$. However, it does not appear as an homogeneous feature although the intensity variations may be due in part to the low count rate. Two particularly bright spots can be observed, one at $\lambda_{I I I} \approx 150^{\circ}$ and relatively low latitude $\left(57^{\circ} \mathrm{N}\right)$, the other at high latitude near the north pole (about $80^{\circ}$ ) between the western limb and $\lambda_{I I I} \approx 100^{\circ}$. Another bright feature, although less intense, is also observed on the $150^{\circ}$ meridian, poleward of the first one (at $\approx 67^{\circ} \mathrm{N}$ ). The count level was determined by fitting a gaussian function to the count rate measured in 20-pixel wide areas across the emission regions. The peak level reaches $1.0 \mathrm{C} \mathrm{pix}^{-1}$ (elementary pixel) in the bright area near the pole and $0.9 \mathrm{C}$ pix $^{-1}$ in the bright spot located near $57^{\circ} \mathrm{N}$ and $\lambda_{I I I}=150^{\circ}$ whereas the mean disk level is about $0.7 \mathrm{C} \mathrm{pix}^{-1}$. The additional count rate of $0.2 \mathrm{C}$ pix $^{-1}$ in the aurora translates into an emission rate at Ly- $\alpha$ (plus some $\mathrm{H}_{2}$ emission) of about $100 \pm 25 \mathrm{kR}$ above the disk background and corresponds to a signal-to-noise ratio of about 3. The uncertainty of about $50 \%$ on the UV filter transmission is another potential source of absolute intensity estimate. Large variations are observed along the oval, with no measurable auroral increase above the background at some longitudes. In this case, the detectability limit set by the noise is estimated to be $\sim 25 \mathrm{kR}$. The FWHM of the gaussian fit on the bright spots is about 1 arcsec corresponding to a latitudinal extension of $\sim 5^{\circ}$ of latitude $(5900 \mathrm{~km})$ for the
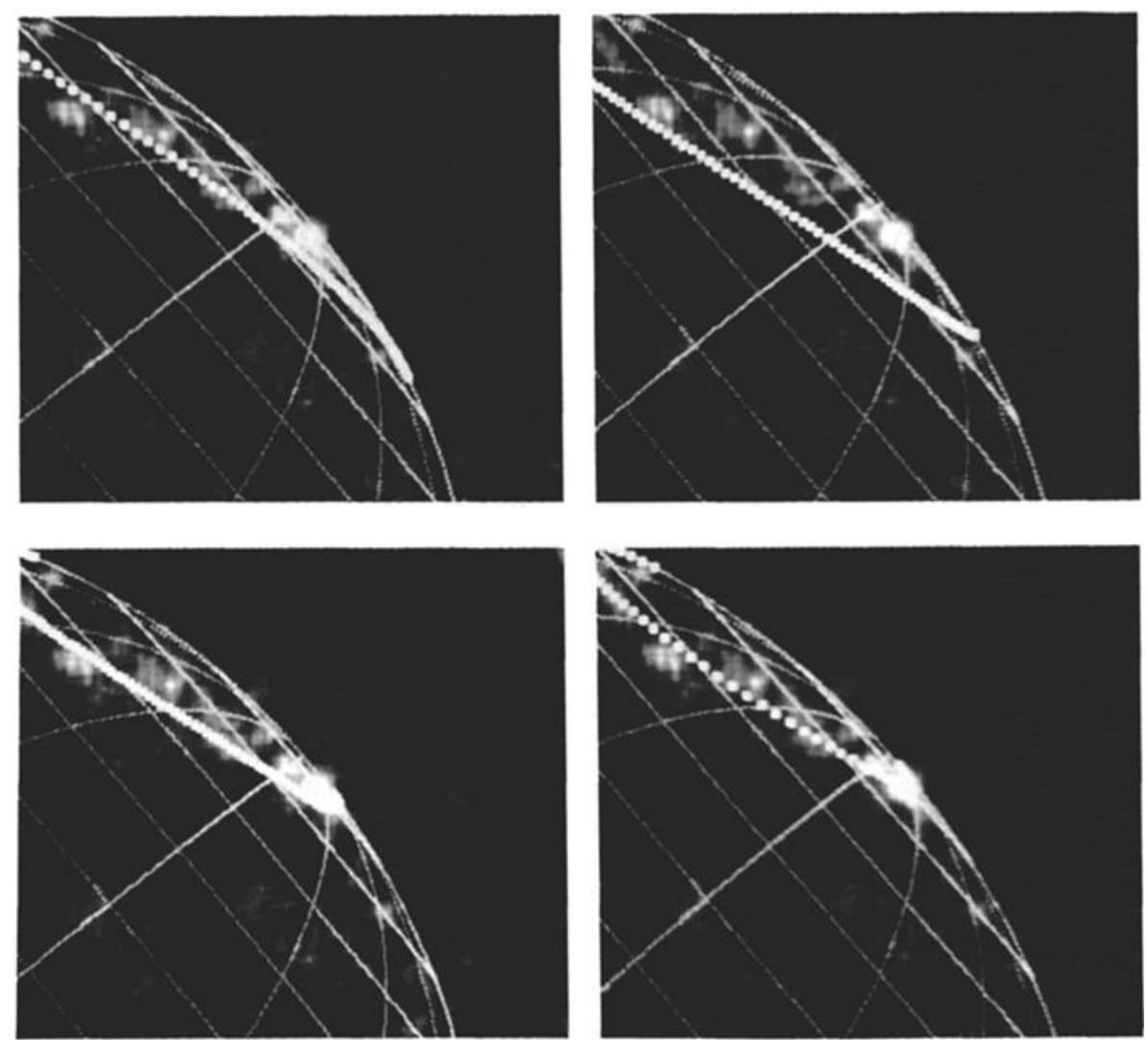

Figure 2: comparison of the Lyman- $\alpha$ aurora of figure 1 with:

(a) top left: the equatorward boundary of the UV auroral zone observed with the spectrometer on board Voyager (Broadfoot et al., 1988);

(b) top right: the footprint of the $\mathrm{L}=6$ magnetic field lines crossing the Io orbit $\left(\mathrm{O}_{4} \mathrm{GSFC}\right.$ model);

(c) bottom left : the footprint of the $\mathrm{L}=15$ magnetosheet field lines $\left(\mathrm{O}_{4}\right.$ GSFC model);

(d) bottom right: the footprint of the $L=30$ field line. 
bright spot at $57^{\circ}$ at the $\mathrm{CML}$ and $\sim 3^{\circ}(3500 \mathrm{~km})$ at $80^{\circ}$ near the limb. The 1 arcsec width may be considered as an upper limit for two reasons. First, the spherically aberrated image of the HST spreads the photons over an area exceeding the actual thickness of the emission region. Although the central core of the point spread function extends over a few pixels in radius, only $50 \%$ of the energy is contained within a circle of 16 pixel radius at $1216 \AA$. Second, the optical thickness of the Lyman- $\alpha$ emission tends to widen the emission and smooth its spatial variations with respect to the precipitation region. The latitudinal extent of the diffuse lower level emission is clearly larger, probably of the order of several arcsec, perhaps covering part of the polar region. However, due to the low signal to noise, and an unfavorable side viewing geometry, it cannot be accurately determined from this single image.

Emission rates previously deduced from the Voyager and IUE spectrometer measurements were intrinsically uncertain due to the lack of information concerning the width of the auroral zone. The best information available so far was gathered when the Voyager UVS aperture scanned north/south across the oval. On some occasions, the polar limb seemed void of emission and the arc seemed to be narrower than the $6000 \mathrm{~km}$ of the slit projection (Sandel et al., 1979). However, the poleward boundary of the "UVS oval" could not be unambiguously detected. Therefore, due to the lack of morphological data, somewhat arbitrary assumptions had to be made to convert the apparent surface brightness detected in the field of view to absolute emission rates. For example, a $60 \mathrm{kR}$ Lyman- $\alpha$ brightness was derived by Sandel et al. (1979) from the Voyager 2 NSMAP scan assuming a width of $6000 \mathrm{~km}$ (mapping along the field line an Io torus confined to $\mathrm{L}=5.9 \pm 1$ ). Similarly, IUE Lyman- $\alpha$ auroral intensities ranging from below instrumental threshold to $42 \mathrm{kR}$ were derived assuming a 2 arcsec north-south extent of the aurora by Clarke et al. (1980). Other intensity estimates (Clarke et al., 1989) were based on the assumption that the IUE small $(3 \mathrm{arcsec})$ or large $(5.5 \mathrm{arcsec} \times 8.9$ arcsec) apertures were filled with auroral emissions and ranged from 5 to $10 \mathrm{kR}$. Our HST image indicates that, if the auroral width observed here is typical, the IUE intensities previously reported should probably be scaled up by a factor of 3 to 9 to account for the 1 arcsec width deduced here. Therefore, the present observations are not incompatible with previous Ly- $\alpha$ intensity estimates.

Figure 2 compares the observed morphology with the location of the Voyager "UVS oval" and with the footprint of several characteristic magnetic field lines in the GSFC $\mathrm{O}_{4}+$ current sheet model (Connerney et al., 1981): $\mathrm{L}=6$ (Io's orbit which is imbedded into the plasma torus region extending between about $\mathrm{L}=5.5$ and 8 , where wave-particle interaction is expected to diffuse the energetic particles into the atmospheric loss cone), $L=15$ (plasmasheet where plasma instabilities such as the tearing instability are likely to occur by analogy with the Earth) and $\mathrm{L}=30$ (so-called open field line, possibly connected to the solar wind). In spite of the low count level, it appears that, in this sector, the $\mathrm{L}=6$ footprint is everywhere equatorward of the HST observations and does not match the Lyman- $\alpha$ satisfactorily, except for the low latitude bright spot at $\lambda_{I I I} \approx 150^{\circ}$, which is just poleward of this oval.

In contrast, the observed aurora is in reasonable agreement with the UVS oval in this longitude sector, although centered a few degrees poleward. It is important to stress that this oval defines the equatorward envelope of the actual UV auroral boundary at the time of the encounter and that most of the UVS emissions were observed several degrees poleward or more. Among the theoretical footprints, a substantially better fit is obtained with the L-shell $=15$ and 30 footprints than with $\mathrm{L}=6$. However, it must be born in mind that in the longitude range of this observation, the $\mathrm{L}=15$ shell oval footprint is not far from the UVS oval. In addition, the fits are globally good for the arc, but the bright spot at $\lambda_{I I I} \approx$ $150^{\circ}$ seems to lie equatorward of the ovals. As for the second spot on the same meridian, it even lies poleward of the 'open field-line' $(\mathrm{L}=30)$ oval.

Unfortunately, the limited accuracy on the location of the limb $(\approx 1$ arcsec) limits the precision of the comparison: a limb shifted by 1 arcsec towards the north would put the $\mathrm{L}=6 \mathrm{O}_{4}$ footprint just equatorward of the observed oval. However, in such a case, a significant segment of the footprint, westward of the high latitude bright spot, would be surprisingly dark. In addition, the magnetic field model itself, deduced from in-situ measurements, is subject to some uncertainty when extrapolated to the surface. This point was discussed by Connemey (1992) and an uncertainty on the order of a fraction of an arcsec can also be expected from this source (see $\mathrm{O}_{6}$ footprint plots in Connerney, 1992). However, the size of the model oval itself is unaffected by the uncertainty. Therefore, an estimate of the relative agreement of the ovals may also be obtained independently of the limb location and of the magnetic field model by comparing the angular extension of the observed emission region, the UVS and theoretical ovals. The observed extension is about 12.9 arcsec, to be compared with 13.2 arcsec for $L=15,16.6$ arcsec for UVS and 17.7 arcsec for $L=6$ ovals. Additional observations, especially at other CMLs (where the ovals are further apart from each other) would be useful to confirm this result.

In contrast to most previous spectrometer observations, no intensity enhancement above the disk mean level is observed with the $\lambda_{I I I} \approx 180^{\circ}$ meridian but a bright spot is observed at a somewhat lower longitude $\left(\lambda_{I I} \approx 150^{\circ}\right)$. In addition, the brightest region is located near the north pole, between the westem limb and $\lambda_{I I I} \approx 100^{\circ}$. This result must be used with caution since this effect might also be due to the nearly tangential viewing of the arc and the subsequent increase of the optical path (limb brightening). It could also be a real intensity maximum possibly related to the intense field-aligned currents and particle beams observed almost simultaneously with this image in the evening sector of the Jovian magnetosphere by several instruments on board the ULYSSES spacecraft (Sanderson, 1992; Balogh et al., 1992). Comparison between these observations and in-situ measurements is in progress.

\section{Discussion}

Infrared $\mathrm{CH}_{4}$ auroral hot spots were also observed poleward of the "UVS oval" (Caldwell et al., 1988) and are statistically coincident with the positive detections of UV aurora by Voyager (Prangé, 1991). They are however restricted to the $\lambda_{I I I} \approx 160^{\circ}-200^{\circ}$ sector, a region possibly associated with harder particle precipitation or enhanced methane concentration above the emission layer (Livengood et al., 1990) where increased absorption of emission shortward of $\sim 1300^{\circ} \mathrm{A}$ is expected. This is precisely the longitude zone where this image shows a relative intensity minimum. On the other hand, recent observations of the ionospheric $\mathrm{H}_{3}^{+}$ emission at $3.53 \mu \mathrm{m}$ (Drossart et al., 1992) exhibit two hot spots in the northern hemisphere located within the belt of UVS auroral emission slightly poleward of the UVS oval. In particular, the peak located near $\lambda_{I I}=150^{\circ}$ lies at the same time along the UVS oval and on open field lines if compared with the theoretical $\mathrm{O}_{4}$ footprints, in agreement with the HST image presented here. Although no firm conclusion can be drawn before a careful analysis of the geometrical and radiative transfer effects is performed on our HST image, we can mention that the images of the $\mathrm{H}_{3}^{+}$aurorae, all recently obtained over a period of one year, also exhibit a relative 
intensity minimum at $\lambda_{I I I}=180^{\circ}$ between two maxima close to $150^{\circ}$ and $220^{\circ}$. Observations of the $\mathrm{H}_{3}^{+}$emission by Baron et al. (1991) which show auroral emission partly extending along the UVS oval, with bright spots around $145^{\circ}$ and $230^{\circ}$, a lower intensity around $180^{\circ}$ and a broad emission region extending around the pole, also present some similarity with this Lyman- $\alpha$ image.

In conclusion, our results provide evidence that monitoring of the Lyman- $\alpha$ aurora, its morphology and intensity variations can be performed from Earth orbit with the Hubble Space Telescope. More observations in different configurations are needed to fully characterize the structure of the Lyman- $\alpha$ aurora in comparison with the Voyager and IUE observations, ground-based infrared images and model predictions. However, the initial results reported here do not conflict with previous auroral measurements in this longitude sector. They indicate that the main auroral process is probably not located at the footprint of the theoretical Io footprint, but rather on high latitude field lines. The structure of the brightest features is patchy, exhibiting large longitudinal inhomogeneities, suggesting that some of them could lie on field lines different from the mean oval (either connected to the Io torus and/or to the polar cap). Additional direct information is obtained on the width of the aurora and the auroral brightness. Specific issues, such as the longitudinal brightness variation will require more thorough analysis and modeling and confirmation by additional observations before they can be physically interpreted.

Acknowledgements. We are very grateful to the STScI moving target scheduling team, especially Alex Storrs for crucial assistance and support throughout the program. We thank G. Munhoven for his assistance in the data analysis and J.E.C. Connerney who provided the magnetic field data. One of the authors (JCG) acknowledges support from the Belgian National Fund for Scientific Research (FNRS). This work was supported in part by the PRODEX ESA program of the Belgian Science Policy Office - Prime Minister's Services. R. Prangé acknowledges support from the CNRS/INSU grant "ATP Planétologie" 803742.

\section{References}

Ajello, J.M., D. Shemansky, T.L. Kwok, and Y.L. Yung, Studies of extreme-ultraviolet emission from the Rydberg series of $\mathrm{H}_{2}$ by electron impact, Phys. Rev., 29, 636-654, 1984.

Balogh, R.J. M.A. Dougherty, M., A. Forsyth, D.J. Southwood, E.J. Smith, B.T. Tsurutani, N. Murphy and M.E. Burton, Magnetic field observation during the Ulysses flyby of Jupiter, Science, 1992 in press.

Baron, R., R.D. Joseph, T. Owen, J. Tennyson, S. Miller, and G.E. Ballester, Imaging Jupiter's aurorae from $\mathrm{H}_{3}^{+}$ emissions in the 3-4 micron band, Nature, 353, 539-542, 1991.

Broadfoot, A.L., M.J.S. Belton and P.Z. Takacs, Extreme ultraviolet from Voyager 1 encounter with Jupiter, Science, 204, 979-982, 1979.
Broadfoot, A.L., B.R. Sandel, D.E. Shemansky, J.C. McConnell, G.R. Smith, J.B. Holberg, S.K. Atreya, T.M. Donahue, D.F. Strobel, and J.L. Bertaux, , Overview of the Voyager ultraviolet spectrometry results through Jupiter encounter, J. Geophys. Res., 86, 8259-8284, 1981.

Caldwell, J.A., R. Halthore., J. Orton, and J. Bergstahl, Infrared polar brightenings on Jupiter IV. Spatial properties of methane emission, Icarus, 74, 331-339, 1988.

Clarke, J.T., H.W. Moos, S.K. Atreya and A.L. Lane, Observations from earth and variability of the polar aurora of Jupiter, Astrophys. J., 241, 179-182, 1980.

Clarke, J.T., J. Trauger, and J.H. Waite, Doppler shifted $\mathrm{H}$ Ly- $\alpha$ emission from Jupiter's aurora, Geophys. Res. Lett., 16, 587-590, 1989.

Connerney, J.E.P., M.H. Acuña, and N.F. Ness, Modeling the Jovian current sheet and inner magnetosphere, Geophys. Res., 86, 8370-8384, 1981.

Connerney, J.E.P., Doing more with Jupiter's magnetic field, In Planetary Radio Emissions III, H.O. Rucker, S.J. Bauer, and M.L. Raise, Eds., Austrian Academy of Science Press, in press.

Drossart, P., R. Prangé, and J.P. Maillard, Morphology of infrared $\mathrm{H}_{3}^{+}$emission in the auroral regions of Jupiter, Icarus, 1992, in press.

Herbert, F., B.R. Sandel, and A.L. Broadfoot, Observations of the Jovian UV aurora by Voyager, J. Geophys. Res., 92, 3141-3154, 1987.

Livengood, T.A., D.F. Strobel, and H.W. Moos, Long-Term study of longitudinal dependence in primary particle precipitation in the north Jovian aurora, J. Geophys. Res., 95, 10375-10383, 1990.

Paresce, F., Faint Object Camera Instrument Handbook, version 3.0, STScI , 1992.

Prangé, R., Jovian UV auroral, IR auroral, and particle precipitations : A common origin ?, Astron. Astrophys., 251, L15-L18, 1991.

Sandel, B.R. et al., Extreme ultraviolet observations from Voyager 2 encounter with Jupiter, Science, 206, 962-966, 1979.

Sanderson, T.R., Energetic particles in the Jovian magnetosphere: Observations of the COSPIN instrument on the Ulysses spacecraft, presented at the XII Magnetospheresplanets "Goertz-Smith Memorial" Symposium, Los Angeles, June 22-26, 1992.

V. Dols and J.C. Gérard, Institut d'Astrophysique, Université de Liège, 5, avenue de Cointe B-4000 Liège - Belgium.

F. Paresce, Space Telescope Science Institute (on assignment from the Astrophysics Division, Space Science Department, ESA), 3700, San Martin Drive, MD 21218, USA.

R. Prangé, Institut d'Astrophysique Spatiale, Bâtiment 121, Université d'Orsay, F-91405, Orsay, France.

A. Vidal-Madjar, Institut d'Astrophysique - CNRS, Boulevard Arago, 98 bis, Paris, F-75014, France.

(received : June 2, 1992;

revised : July 17, 1992;

accepted : August 20, 1992.) 